\title{
Le numérique pour l'apprentissage collaboratif : nouvelles interfaces, nouvelles interactions
}

\author{
Digital technology for collaborative learning: \\ new interfaces, new interactions
}

\section{Entornos digitales para el aprendizaje colaborativo: nuevas interfaces, nuevas interacciones}

RESPONSABLES DU NUMÉRO :

Stéphanie Fleck, maître de conférences

Université de Lorraine, France

stephanie.fleck@univ-lorraine.fr

Luc Massou, maître de conférences

Université de Lorraine, France

luc.massou@univ-lorraine.fr

\begin{abstract}
RÉSUMÉ
Les systèmes éducatifs, de l'école à l'université, sont confrontés à un contexte de profonds changements qui transforment les pratiques d'enseignement et l'apprentissage formel. Avec l'ambition de permettre aux apprenants d'apprendre, de faire, d'être et de décider individuellement et collectivement dans un monde complexe en perpétuel mouvement, les institutions encouragent la mise en œuvre d'approches collaboratives. Au cœur de ces enjeux, les technologies numériques voient également leur diversité s'accroître rapidement. Les interfaces de nouvelle génération, telles que les interfaces tangibles, de réalité mixte ou robotiques, modifient les interactions avec les autres et les connaissances en offrant des paradigmes d'interaction homme-machine différents de ceux offerts par les interfaces écranclavier-souris. L'objectif de ce cinquième numéro thématique, délibérément interdisciplinaire, est de s'interroger sur l'impact et la place des technologies numériques - y compris celles de
\end{abstract}


nouvelle génération - pour l'apprentissage collaboratif. Les interactions avec le savoir, avec les autres au sein des communautés d'apprentissage ou d'enseignement, et les systèmes numériques eux-mêmes sont tour à tour au centre des problématiques des auteurs de ce numéro. Leurs douze contributions, par des approches complémentaires, enrichissent le corpus de connaissances empiriques et méthodologiques. Ils examinent les approches collaboratives entre enseignants, l'engagement à collaborer entre apprenants en présentiel ou à distance, la construction de l'apprentissage qui en résulte, et l'influence des systèmes sur ces derniers. Cela permet d'identifier plusieurs pistes à approfondir dans les travaux à mener sur cette thématique des nouvelles interfaces et interactions numériques pour l'apprentissage collaboratif.

Mots-clés : collaboration, interactions, interfaces, apprentissage, numérique en éducation

ABSTRACT

Education systems, from school to university, are facing a context of profound changes that are transforming teaching practices and formal learning. With the ambition of enabling everyone to learn, do, be, and decide, individually and collectively, in a complex world in perpetual motion, institutions encourage the implementation of collaborative approaches. At the heart of these challenges, digital technologies are also seeing their diversity expand rapidly. New-generation interfaces such as tangible, mixed reality, or robotic interfaces modify interactions with others and knowledge by offering alternative human-computer interaction paradigms to those offered by screen-keyboard-mouse interfaces. The objective of this fifth thematic issue, deliberately interdisciplinary, is to question the impact and place of digital technology - including new-generation ones - in collaborative learning. Interactions with knowledge, with others within learning or teaching communities, and digital systems themselves are in turn at the center of the authors' focus in this issue. Their twelve contributions, through complementary approaches, increase the corpus of empirical and methodological knowledge. They examine collaborative approaches among teachers, the commitment to collaboration among learners in face-to-face or distance learning, and the resulting construction of learning, in light of the systems' influence on it. This allows identifying several directions for further work on new digital interfaces and interactions for collaborative learning.

Keywords: collaboration, interactions, interfaces, learning, digital education

\section{RESUMEN}

Los sistemas educativos, desde la escuela hasta la universidad, se enfrentan a un contexto de profundos cambios que están transformando las prácticas de enseñanza y el aprendizaje formal. Con la ambición de permitir que los alumnos aprendan, hagan, sean y decidan, individual y colectivamente, en un mundo complejo en perpetuo movimiento, las instituciones alientan la aplicación de enfoques de colaboración. En el centro de los desafíos, las tecnologías digitales también están viendo cómo su diversidad se expande rápidamente. Las interfaces de nueva generación, como las interfaces tangibles, de realidad mixta o robóticas, modifican las interacciones con los demás y con el conocimiento, ofreciendo paradigmas de interacción hombre-computadora alternativos a los que ofrecen las interfaces pantalla- 
teclado-ratón. El objetivo de este quinto número temático, que es deliberadamente interdisciplinario, es cuestionar el impacto y el lugar de la tecnología digital —incluida la de nueva generación- para el aprendizaje en colaboración. Las interacciones con el conocimiento, con otros dentro de las comunidades de aprendizaje o enseñanza, y los propios sistemas digitales están en el centro de los intereses de los autores de este número. Sus doce contribuciones, a través de enfoques complementarios, aumentan el corpus de conocimiento empírico y metodológico. Examinan los enfoques de colaboración entre los profesores, el compromiso de colaboración entre los alumnos en la enseñanza presencial o a distancia, la consiguiente construcción del aprendizaje y la influencia de los sistemas en dichos alumnos. Esto permite identificar varias direcciones para futuros trabajos en este tema de nuevas interfaces digitales e interacciones para el aprendizaje colaborativo.

Palabras clave: colaboración, interacciones, interfaces, aprendizaje, educación digital

Les politiques éducatives actuelles placent l'école et l'université dans un mouvement de transformation et d'innovation pédagogiques dans lequel le numérique est généralement considéré comme un des leviers prioritaires. Or, les interactions avec le numérique sont à l'aube d'un changement profond porté par des avancées technologiques rapides, dont les interfaces mobiles, tactiles et connectées (tablettes, smartphones, objets connectés) ne sont que les toutes premières représentantes. Ces mutations hors l'école creusent progressivement l'écart avec les pratiques en contexte scolaire, où la manipulation de matériel numérique demeure très variable selon les contextes locaux et internationaux. Parallèlement, les programmes d'éducation et de formation partout dans le monde, en lien avec les recommandations de l'OCDE, demandent le développement non seulement de connaissances, mais aussi de compétences transversales. Ils renforcent la nécessité de la mise en place de conduites de projets par les élèves et les étudiants, leur demandant de travailler en équipe, de partager des tâches ou de s'engager dans un dialogue constructif (Gibert, 2018). L'école et la classe deviennent alors des lieux de collaboration, d'entraide et de mutualisation des savoirs (Baudrit, 2007; Lessard et al., 2009).

Dans ce contexte scolaire, auquel est venue s'ajouter la crise sanitaire due à la COVID-19, les interactions générées par les environnements numériques sont à interroger, car elles peuvent constituer un élément d'accompagnement, de renforcement, de facilitation, voire d'innovation pour l'apprentissage collaboratif en modifiant les relations sociales (échanges entre pairs, travail en groupes) et les modalités de partage et de construction des connaissances (computer supported collaborative learning: Stahl et al., 2006). Elles s'interrogent sur les apprentissages et la transformation des pratiques enseignantes en modifiant notamment l'écosystème de la classe et les modalités d'interactions avec les savoirs (Dupriez, 2010). Elles peuvent également s'appuyer sur des interfaces numériques de nouvelle génération modifiant les modalités d'interactions humain-machine, mais aussi entre leurs utilisateurs et avec les représentations des savoirs. C'est notamment le cas pour celles qui exploitent la réalité augmentée, les objets connectés, les robots et/ou les dispositifs tactiles ou tangibles (Cuendet et al., 2013; Fleck et Hachet, 2016, Schneider et al., 2011; Kubicki et al., 2014). Ces interfaces de nouvelle génération en milieu scolaire, alternatives des écrans-clavier-souris, remettent en question les attentes, les besoins et les réalités de pratiques, non seulement des élèves mais aussi de leurs enseignants, et plus largement de toute la communauté éducative. Elles peuvent également favoriser les démarches de conception participative (co-design, Fab Lab à l'école, living lab éducatif) impliquant élèves et enseignants, et permettre à ces derniers de changer de rôle ou de posture durant ces activités de travail collectif. 
L'objectif de ce numéro thématique est ainsi d'interroger l'impact et la place du numérique - dont les interfaces de nouvelle génération - pour l'apprentissage collaboratif, en s'appuyant sur des recherches empiriques menées en contexte scolaire et en les situant par rapport aux travaux scientifiques déjà existants : quelles interactions (humain-machine, entre pairs, avec les savoirs)? quelles interfaces? quelles configurations du travail collectif? quelles modalités d'apprentissage? quelles évolutions dans les pratiques enseignantes? Pour proposer une lecture davantage transversale des douze contributions de ce numéro thématique, nous pouvons dégager plusieurs axes de questionnement présents dans les différentes catégories de textes retenus :

- $\quad$ l'influence des interfaces numériques - qu'elles soient de nouvelle génération ou pas - sur les apprentissages collaboratifs et sur l'engagement dans la collaboration entre apprenants;

- les typologies de pratiques collaboratives chez les enseignants et les enjeux et dynamiques professionnels qui y sont associés.

Dans le premier axe, plusieurs contributions interrogent selon des angles complémentaires l'impact pédagogique d'outils numériques sur les pratiques et/ou apprentissages collaboratifs à distance chez les élèves et les étudiants. Fierro Porto et Schofield analysent ainsi le fonctionnement d'un dispositif de formation d'anglais en ligne sur la plateforme Moodle intégrant les pratiques informelles des étudiants, en fondant le choix des ressources, contenus et activités sur ce que les étudiants font habituellement en dehors des cours et en mettant l'accent sur ce qu'ils peuvent apporter du fait de leur familiarité avec les éléments du dispositif numérique mis en place. Les apports du numérique et du distanciel concernent ici la communication asynchrone médiée par ordinateur et les opportunités d'apprentissage collaboratif qui donnent lieu à des interactions étudiantes inédites au sein desquelles les apprenants ont le rôle d'experts de leur domaine d'études et de connaisseurs des contenus proposés, car liés à leurs propres pratiques en dehors des cours. Le texte de Marty et Thomas Vasquez propose une démarche ethnographique pour étudier un dispositif hybride d'aide centré sur la réussite et l'intégration des étudiants en première année de Licence à Montpellier. II prend appui sur le recueil de matériaux de terrain (storyboards pédagogiques, journal de bord regroupant les observations réalisées dans le cadre du dispositif, comptes rendus de séances et de réunions pédagogiques) afin d'explorer in vivo les rouages de ce dispositif pédagogique pensé en blended learning (Bower et al., 2014). Les résultats obtenus montrent que l'hybridation mise en œuvre facilite ce type de pédagogie par projet et la mise en place de collaborations plurielles souvent inusitées en première année de Licence : entre étudiants, entre étudiants et « étudiants-parrains ", avec l'enseignant, avec des partenaires du projet. Dans un autre registre, l'article de praticiens rédigé par Deschênes et Tremblay prend appui sur une activité de coélaboration de connaissances mise en place auprès de six étudiants en sciences de l'éducation dans le cadre d'un cours universitaire, sur le thème de l'équité numérique. Pour cela, elles ont utilisé plusieurs outils numériques en présentiel et en ligne, dont le Knowledge Forum : forum à structure neuronale et non linéaire, qui permet de créer un réseau de contributions individuelles et d'« échafaudages » davantage collaboratif (pour réaliser une tâche avec l'aide des autres). Leur étude de cas permet d'identifier les processus de régulation mobilisés (autorégulation, corégulation et régulation partagée), les affordances des outils numériques et les influences mutuelles des contextes de coélaboration comme des facteurs clés d'engagement dans la collaboration. Enfin, le texte de Meunier développe une discussion autour de l'approche instrumentale d'un débat en ligne (Lonchamp, 2012; Rabardel, Beguin, 2005) fondée sur une pluralité des formes de médiation liées aux activités instrumentées via le numérique : épistémique, pragmatique, interpersonnelle et réflexive. L'un des principaux intérêts de la notion d'instrument pour l'analyse des situations d'enseignement collaboratif réside dans la prise en compte d'un double mouvement : la découverte des propriétés de celui-ci et l'adaptation des trois types de schèmes concernés (schèmes d'utilisation, d'action et d'activité collectives). Les schèmes d'activité collective concernés par son étude sont de trois ordres : se mettre d'accord sur le choix de l'outil, sur le mode de gestion des versions du document commun et sur le processus d'arbitrage entre les différentes propositions soumises. 
Trois textes questionnent davantage l'évaluation des qualités ergopédagogiques des interfaces numériques elles-mêmes et de leurs influences sur les usages pédagogiques. L'article de Baraudon, Lanfranchi, Bastien et Fleck se positionne tout d'abord sur un outillage méthodologique original pour rendre possible l'évaluation de l'utilisabilité des interfaces numériques par les jeunes apprenants eux-mêmes. Leur constat préalable est que les interfaces numériques se diversifient actuellement très rapidement et vont parfois au-delà des interactions Windows, Icons, Menus and Pointing device (WIMP) ou de la saisie tactile, chacune possédant ainsi leurs propres paradigmes d'interactions. II peut s'agir, par exemple, de robots éducatifs (Frei et al., 2000; Sapounidis et al., 2019), d'interfaces utilisateur tangibles associées ou non à de la réalité mixte (Bumbacher et al., 2013; Fleck et al., 2015). Cette diversité d'interfaces et d'interactions homme-machine associées peut influencer les méthodes de travail des enfants et affecter potentiellement leur développement et la qualité de leurs apprentissages. Leur solution repose ainsi sur la validation d'une échelle de mesure d'évaluation de l'utilisabilité, telle que perçue par les jeunes utilisateurs (9 à 11 ans), en interrogeant trois dimensions : la performance du système, la satisfaction lors de l'utilisation, et la " learnability » définie par Nielsen (1994), c'est-à-dire la facilité de prise en main de l'interface. Deux autres textes proposent ensuite des retours d'expérience liés à l'usage d'outils numériques en classe ou en ligne. Redouani présente ainsi les résultats de son analyse d'impact de l'usage du tableau blanc interactif (TBI) sur les pratiques pédagogiques dans trois écoles élémentaires de Strasbourg (France), à partir d'entretiens avec les enseignants et d'observations en classe. II considère le TBI comme un dispositif techno-sémio-pragmatique au sens de Peraya (1999), c'est-à-dire qu'il n'apparaît plus comme un outil isolé des autres outils présents dans la classe, mais comme un dispositif qui assure l'interaction entre des variables techniques, symboliques et humaines afin de favoriser la communication dans la salle de classe à travers des interactions susceptibles d'instaurer un apprentissage collaboratif. Ses résultats sont plutôt mitigés, le TBI demeurant surtout un outil de présentation. Dans leur texte de praticiens, Le Thi Kim Oanh et Szilas exposent les enseignements tirés d'une médiatisation et rescénarisation à la volée et en urgence d'une activité de prototypage papier pour la conception de jeux vidéo, qui a basculé brutalement d'un mode présentiel à un mode distanciel en raison de la pandémie en 2020. Cette médiatisation d'une collaboration initialement tangible et présentielle vers la distance s'est réalisée sans possibilité de repenser l'ensemble du scénario pédagogique préalable. Les critères de choix des outils numériques retenus pour cela ont donc été triples : l'utilisabilité, l'expressivité et la conscience de l'autre comme co-manipulateur.

Un autre axe aborde la question centrale dans de nombreux travaux de l'adéquation des interfaces numériques de nouvelle génération aux tâches d'apprentissage, aux profils d'apprenants et aux compétences visées (Bates, 2017; Moreno, 2006; Sauvé, 2010; Wang et al., 2018). Lewis, Plante et Lemire proposent ainsi une revue de littérature sur la pertinence, l'efficacité et les principes pédagogiques de la réalité virtuelle et augmentée en contexte scolaire à partir d'une sélection de 29 articles publiés depuis 2017 sur ce thème : quelles oppositions? quelles validations? quels concepts et paradigmes utilisés? quels avantages et limites identifiés chez ces artefacts numériques? Leur étude bibliographique les amène plus particulièrement à évaluer leur pertinence et leur efficacité pédagogiques selon quatre dimensions ou tâches : la motivation des élèves, l'immersion, la collaboration et la conception. Ils en concluent qu'ils peuvent améliorer l'attention et la cognition, mais doivent inclure non seulement des méthodes d'instructions adaptées aux publics cibles, mais aussi utiliser un média approprié pour le domaine de connaissance de la formation visée. L'entretien mené par Fleck et Massou avec Bétrancourt sur sa conception des ressources numériques pédagogiques dans une perspective cognitive et ergonomique, et sur les usages des technologies numériques de nouvelle génération (tactiles, tangibles, connectées) dans différents contextes de formation et d'enseignement conduit à la même conclusion : la chercheuse, directrice de l'unité de Technologies de formation et d'apprentissage (TECFA) à l'Université de Genève (Suisse), y souligne l'importance de la congruence technopédagogique des nouvelles interactions sensorimotrices avec les exigences de la tâche d'apprentissage et du contexte scolaire dans lequel elles sont utilisées afin de mieux en évaluer les apports réels (Bétrancourt, Bozelle, 2012). 
Le dernier axe correspond à trois textes davantage axés sur les pratiques collaboratives des enseignants avec le numérique et leur impact en termes d'enjeux professionnels. Le texte de Massou, Lanfranchi, Chauvel et Fleck propose ainsi une typologie des pratiques de travail collectif entre pairs et en classe, avec ou sans numérique, issu d'une enquête régionale menée en ligne (Massou et al., 2019) auprès d'enseignants de cycles 3 et 4 (écoles et collèges) du département de la Moselle (France). Au travers de l'analyse des 972 réponses obtenues, l'étude statistique permet d'identifier quatre catégories de variables associées aux pratiques de travail collectif chez les enseignants, qui s'agrègent en clusters selon deux axes paradigmatiques : enseignant vs apprenant, autocentré sur la classe vs hétérocentré. D'autre part, elle permet d'analyser la force de prédiction de six catégories de variables du travail en groupe avec leurs élèves : les aspects sociodémographiques et professionnels; l'attitude sur les technologies du numérique et leurs usages; les types de collaboration avec les collègues; les types d'activité de travail en groupe; l'instrumentalité perçue du travail en groupe pour favoriser l'émergence de compétences; les ressources matérielles et numériques mises à disposition des enseignants. Parmi les résultats significatifs obtenus figure l'impact des usages d'outils et ressources numériques qui s'avère finalement très secondaire dans les catégories et facteurs identifiés. Dans un terrain similaire, Ferone et Crinon ont étudié la communauté formée en ligne dans le réseau Twictée, qui regroupe plusieurs centaines d'enseignants francophones de l'école élémentaire et du collège mettant en œuvre un dispositif innovant d'enseignement de l'orthographe et échangeant à distance sur cet enseignement. À partir d'entretiens menés avec ces enseignants, ils se demandent si ces communautés sont censées favoriser des formes de réflexion et d'auto-analyse plus approfondies qu'en présence grâce à la confrontation des points de vue et aux échanges écrits, alors que la réalité est parfois bien différente (Bergviken Rensfeldt, Hillman et Selwyn, 2018). Leur étude permet de décrire les contours d'une communauté d'apprentissage professionnelle avec quatre profils de participants (selon la nature et l'intensité de leur engagement) et d'identifier des dynamiques de changement ponctuelles dans leurs pratiques : modes d'organisation pédagogique, intégration du numérique dans leur enseignement, changements identitaires. Enfin, ces enjeux professionnels sont également au cœur du texte de Brandl sur les collaborations interprofessionnelles menées autour d'un projet d'innovation pédagogique, à l'université cette fois. Son analyse sociologique et qualitative par entretiens et observations permet de comprendre les dispositions socioprofessionnelles des individus impliqués, entendues comme structures cognitives individuelles qui orientent ou déterminent les actions dans un contexte donné et qui sont incorporées dans un processus de socialisation professionnelle (Berger et Luckmann, 2018) en intériorisant des valeurs, normes et règles d'un champ institutionnel spécialisé (Dubar, 1991). Selon l'auteur, l'innovation pédagogique autorise l'espoir d'une remise en question de certains rapports de force et de croyances jusqu'alors instituées (Lemieux, 2007) et d'inventer de nouveaux dispositifs organisationnels et techniques permettant de contraindre différemment l'ordre actuel des collaborations socioprofessionnelles à l'université.

Pour conclure, les différentes contributions de ce numéro très riche nous permettent d'identifier plusieurs pistes à approfondir dans les travaux à mener sur cette thématique des nouvelles interfaces et interactions numériques pour l'apprentissage collaboratif. Tout d'abord, et comme le soulignent Ferone et Crinon dans leur conclusion, il apparaît nécessaire de pouvoir observer les dynamiques de ces collectifs - chez les apprenants et chez les enseignants - sur la durée (Beauné et al., 2019), mais aussi celles des trajectoires individuelles de leurs membres. Dans le même ordre d'idées, il sera intéressant de s'appuyer sur les résultats des études majoritairement qualitatives proposées ici, et qui dépendent de leurs terrains respectifs, pour les combiner avec des approches quantitatives plus larges qui permettent de dépasser le niveau micro de certains contextes scolaires. Ce niveau demeure nécessaire et incontournable pour ce type d'études menées sur des innovations pédagogiques et numériques ciblées, mais les dépasser permettrait d'atteindre davantage le niveau méso d'un établissement ou d'une académie régionale, voire le niveau macro d'un territoire éducatif encore plus large afin d'évaluer leur impact - ou non - sur des transformations de pratiques d'enseignement et d'apprentissage à plus large échelle. Enfin, et comme le suggère Meunier dans son texte de discussion-débat en évoquant des « systèmes instrumentalisables par 
les apprenants » (Lonchamp, 2012), remettre en question et accompagner la place des apprenants dans ces dispositifs numériques pour l'apprentissage collaboratif demeure fondamental afin de renforcer leur autonomie et en les laissant s'emparer à leur façon de ces dispositifs plus ou moins innovants.

\section{Liste de références}

Bates, T. (2017). Virtual reality and education: some thoughts. Online Learning and Distance Education Ressouces, 27 juillet 2017. Récupéré du site https://www.tonybates.ca/2017/07/27/virtual-reality-and-education-some-thoughts/

Baudrit, A. (2007). L'apprentissage collaboratif : plus qu'une méthode collective? De Boeck Université.

Beauné, A., Levoin, X., Bruillard, É., Quentin, I., Zablot, S., Carton, T., Rouvet-Song. C., Normand-Assadi. S., Le Roy. M., Nikishina, T., Mas-Costesèque. S. et Louis Baron, G. (2019). Collectifs en réseau d'enseignants producteurs de ressources. Rapport scientifique des laboratoires STEF et EDA dans le cadre de la convention DNE [rapport de recherche] Université Paris 5 Sorbonne Descartes; ENS Cachan. https://hal.archives-ouvertes.fr/hal-02022830

Berger, P. et Luckmann, T. (2018) La construction sociale de la réalité. Armand Colin.

Bergviken Rensfeldt, A., Hillman, T. et Selwyn, N. (2018). Teachers 'liking' their work? Exploring the realities of teacher Facebook groups. British Educational Research Journal, 44(2), 230-250.

Bétrancourt, M. et Bozelle, C. (2012). Les MITIC au service de la pédagogie spécialisée : mieux connaître leur spécificité pour développer des usages éducatifs pertinents. Revue de Pédagogie spécialisée, 4, 15-23.

Bower, M., Dalgarno, B., Kennedy, G., Lee, M. J. W., Kenney, J. (2014). Blended Synchronous Learning: A handbook for Educators. Australian Government Office for Learning and Teaching. http://www2.curtin.edu.au/cli/local/docs/ID11 1931 Bower Report handbook 2014.pdf

Bumbacher, E., Deutsch, A., Otero, N. et Blikstein, P. (2013). BeatTable: a tangible approach to rhythms and ratios. Communication présentée lors de la $12^{\mathrm{e}}$ conférence internationale sur l'interaction le design et les enfants (IDC), New York, USA.

Cuendet, S., Bonnard, Q., Do-Lenh, S. et Dillenbourg, P. (2013). Designing augmented reality for the classroom. Computers \& Education, 68, 557-569. https://doi.org/10.1016/j.compedu.2013.02.015

Dubar, C. (1991) La socialisation. Construction des identités sociales et professionnelles, Armand Colin.

Dupriez, V. (2010). Le travail collectif des enseignants : au-delà du mythe. Travail et formation en éducation, 7. Récupéré de : http://journals.openedition.org/tfe/1492

Fleck, S., Hachet, M. et Bastien, J. M. C. (2015, June 21-24). Marker-based augmented reality: Instructional-design to improve children interactions with astronomical concepts. Paper presented at the ACM SIGCHI $14^{\text {th }}$ International Conference on Interaction Design and Children, Boston, MA, USA.

Fleck, S. et Hachet, M. (2016). Making tangible the intangible: Hybridization of the real and the virtual to enhance learning of abstract phenomena. Frontiers in ICT, 3, 30.

Frei, P., Su, V., Mikhak, B. et Ishii, H. (2000). Curlybot: designing a new class of computational toys. Communication présentée à la conférence sur les facteurs humains dans les systèmes informatiques SIGCHI, New York, USA.

Gibert, A-F. (2018). Le travail collectif enseignant, entre informel et institué. Dossier de veille de l'IFÉ, 124. Lyon : ENS de Lyon. Récupéré de : http://veille-et-analyses.ens-lyon.fr/DA/detailsDossier.php?\&dossier=124\&lang=fr

Kubicki, S., Pasco, P. et Arnaud, I. (2014). Utilisation en classe d'un jeu sérieux sur table interactive avec objets tangibles pour favoriser l'activité des élèves : une évaluation comparative en cours préparatoire. STICEF, 21(1).

Lemieux, C. (2007) À quoi sert l'analyse des controverses? Société d'études soréliennes, 25, 191-212.

Lessard, C., Kamanzi, P. et Larochelle, M. (2009). De quelques facteurs facilitant l'intensification de la collaboration au travail parmi les enseignants : le cas des enseignants canadiens. Éducation et sociétés, 23(1), 59-77.

Lonchamp, J. (2012). An instrumental perspective on CSCL systems. International Journal of Computer-Supported Collaborative Learning, 7(2), 211-237. 
Massou, L., Morelli, P, Kellner, C., Fleck, S., Lanfranchi, J.-B., Bastien, C. et Chauvel, B. (2019). Travail collectif en classe et numérique : quelles pratiques? quels facteurs facilitateurs? Poster présenté au colloque e-Fran Enseigner et apprendre : les apports de la recherche. Méthodes et premiers résultats, octobre, Paris, France. Récupéré de : https://hal.univ-lorraine.fr/hal-02395201/document

Moreno, R. (2006). Learning in high-tech and multimedia environments. Current Directions in Psychological Science, 15(2), 63-67.

Nielsen, J. (1994). Usability engineering. Morgan Kaufmann Publishers Inc.

Peraya, D. (1999). Médiation et médiatisation : le campus virtuel. Hermès, 25, 153-167.

Rabardel, P. et Beguin, P. (2005). Instrument mediated activity: From subject development to anthropocentric design. Theoretical Issues in Ergonomics Science, 6(5), 429-461.

Sapounidis, T., Demetriadis, S., Papadopoulos, P. M. et Stamovlasis, D. (2019). Tangible and graphical programming with experienced children: A mixed methods analysis. International Journal of Child-Computer Interaction, 19, 67-78.

Sauvé, L. (2010). Les jeux éducatifs efficaces. Dans L. Sauvé et D. Kaufman (dir.), Jeux et simulations éducatifs (p. 43-72). Québec (Québec) : Presses de l'Université du Québec.

Schneider, B., Jermann, P., Zufferey, G. et Dillenbourg, P. (2011). Benefits of a tangible interface for collaborative learning and interaction. Learning Technologies, 4(3), 222-232.

Stahl, G., Koschmann, T. et Suthers, D. (2006). Computer-supported collaborative learning: An historical perspective. Dans R. K. Sawyer (dir.), Cambridge handbook of the learning sciences. Cambridge, UK: Cambridge University Press, 409-426.

Wang, M., Callaghan, V., Bernhardt, J., White, K. et Pena-Rios, A. (2018). Augmented reality in education and training: pedagogical approaches and illustrative case studies. Journal of Ambient Intelligence and Humanized Computing, 9, 1391-1402 